\title{
A BENCHMARK FOR FAULT TOLERANT FLIGHT CONTROL EVALUATION
}

\author{
H. Smaili ${ }^{1}$, J. Breeman ${ }^{1}$, T. Lombaerts ${ }^{2}$, and O. Stroosma ${ }^{2}$ \\ ${ }^{1}$ National Aerospace Laboratory NLR \\ 2 Anthony Fokkerweg, Amsterdam 1059 CM, The Netherlands \\ ${ }^{2}$ Delft University of Technology (DUT) \\ 1 Kluyverweg, Delft 2629 HS, The Netherlands
}

\begin{abstract}
A large transport aircraft simulation benchmark (REconfigurable COntrol for Vehicle Emergency Return - RECOVER) has been developed within the GARTEUR (Group for Aeronautical Research and Technology in Europe) Flight Mechanics Action Group 16 (FM-AG(16)) on Fault Tolerant Control (2004-2008) for the integrated evaluation of fault detection and identification (FDI) and reconfigurable flight control strategies. The benchmark includes a suitable set of assessment criteria and failure cases, based on reconstructed accident scenarios, to assess the potential of new adaptive control strategies to improve aircraft survivability. The application of reconstruction and modeling techniques, based on accident flight data, has resulted in high-fidelity nonlinear aircraft and fault models to evaluate new Fault Tolerant Flight Control (FTFC) concepts and their real-time performance to accommodate in-flight failures.
\end{abstract}

\section{INTRODUCTION}

Fault tolerant flight control allows improved survivability and recovery from adverse flight conditions induced by faults, damage, and associated upsets. This can be achieved by "intelligent" utilization of the control authority of the remaining control effectors in all axes consisting of the control surfaces and engines or a combination of both. In this technique, control strategies are applied to restore stability and maneuverability of the vehicle for continued safe operation and a survivable landing.

From 2004-2008, a research group on Fault Tolerant Control, comprizing a collaboration of 13 European partners from industry, universities, and research institutions:

QinetoQ, Bedford, United Kingdom; 
Airbus, Toulouse, France;

National Aerospace Laboratore (NLR), Amsterdam, The Netherlands;

Deutsches Zentrum für Luft- und Raumfahrt (DLR), Braunschweig and Oberpfaffenhofen, Germany;

Defence Science and Technology Laboratory (DSTL), Bedford, United Kingdom;

Centro Italiano Ricerche Aerospaziali (CIRA), Capua, Italy;

Delft University of Technology, Delft, The Netherlands;

Cambridge University, Cambridge, United Kingdom;

Aalbord University, Esbjerg, Denmark;

University of Lille, Lille, France;

University of Hull, Hull, United Kingdom;

University of Bordeaux, Bordeaux, France; and

University of Leicester, Leicester, United Kingdom

was established within the framework of the GARTEUR cooperation program. The aim of the research group, FM-AG(16), is to demonstrate the capability and potential of innovative reconfigurable flight control algorithms to improve aircraft survivability. The group facilitated the proliferation of new developments in fault tolerant control design within the European aerospace research and academic community towards practical and real-time operational applications. This addresses the need to improve the resilience and safety of future aircraft and aiding the pilot to recover from adverse conditions induced by (multiple) system failures and damage that would, otherwise, be potentially catastrophic. Up till now, faults or damage on board of aircraft have been accommodated by hardware design using duplex, triplex, or even quadruplex redundancy of critical components. The approach of the GARTEUR research is to focus on providing redundancy by means of new adaptive control law design methods to accommodate (unanticipated) faults and/or damage that dramatically change the configuration of the aircraft. These methods take into account a novel combination of robustness, reconfiguration, and (real-time) adaptation of the control laws $[1,2]$.

The potential of the developed FTFC methods to improve aircraft survivability, for both manual and automatic flight, has been demonstrated in 2008 during a piloted assessment in the SIMONA research flight simulator of the Delft University of Technology $[2,3]$. 


\section{DAMAGE TOLERANT FLIGHT CONTROL}

An increasing number of measures are currently being taken by the international aviation community to prevent Loss Of Control In-Flight (LOC-I) accidents due to failures, damage, and upsets for which the pilot was not able to recover successfully despite the available performance and control capabilities. Recent airliner accident and incident statistics [4] show that about $16 \%$ of the accidents between the 1993 and 2007 period can be attributed to LOC-I, caused by a piloting mistake, technical malfunction, or unusual upsets due to external (atmospheric) disturbances. However, worldwide civil aviation safety statistics indicate that today 'in-flight loss of control' has become the main cause of aircraft accidents (followed by 'controlled flight into terrain' (CFIT)). Data examined by the international aviation community shows that, in contrast to CFIT, the share of LOC-I occurrences is not significantly decreasing. The actions taken by the aviation community to lower the number of LOC-I occurrences not only include improvements in procedures training and human factors, but also finding measures to better mitigate system failures and increase aircraft survivability in case of an accident or degraded flight conditions.

Adaptive or reconfigurable flight control strategies might have prevented the loss of two Boeings 737 due to a rudder actuator hard over $[5,6]$ and of a Boeing 767 due to inadvertent asymmetric thrust reverser deployment [7]. The 1989 Sioux City DC-10 incident [8] is an example of the crew performing their own reconfiguration using asymmetric thrust from the two remaining engines to maintain limited control in the presence of total hydraulic system failure. The crash of a Boeing 747 freighter in 1992 near Amsterdam, the Netherlands, following the separation of the two right-wing engines [9], was potentially survivable given adequate knowledge about the remaining aerodynamic capabilities of the damaged aircraft [10]. New kinds of threats within the aviation community have recently been introduced by deliberate hostile attacks on both commercial and military aircraft. A surface-to-air missile (SAM) attack has recently been demonstrated to be survivable by the crew of an Airbus A300B4 freighter performing a successful emergency landing at Baghdad International Airport after suffering from complete hydraulic system failures and severe structural wing damage (Fig. 1).
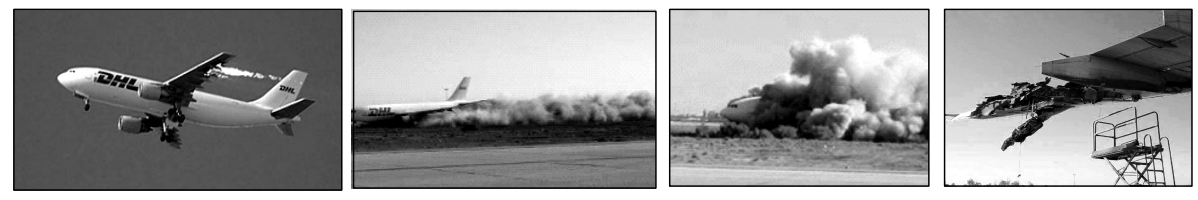

Figure 1 Emergency landing sequence using engines only and left wing structural damage due to SAM missile impact, DHL A300B4-203F, Baghdad, 2003 
Apart from system failures and hostile actions against commercial and military aircraft, recent incident cases also show the destructive impact of hazardous atmospheric weather conditions on the structural integrity of the aircraft. In some cases, clear air turbulence (CAT) has resulted in aircraft incurring substantial structural damage, and loss of engines due to CAT.

Studies of airliner LOC-I accidents $[2,10]$ show that better situational awareness or guidance would have recovered the impaired aircraft and improved survivability if unconventional control strategies were used. In some of the cases studied, the crew was able to adapt to the unknown degraded flying qualities by applying control strategies (e.g., using the engines effectors to achieve stability and control augmentation) that are not part of any standard airline training curriculum.

The results of an LOC-I study concerning the 1992 Amsterdam accident case [10], in which a detailed reconstruction and simulation of the accident flight was conducted based on the recovered Digital Flight Data Recorder (DFDR), formed the basis for realistic and validated aircraft accident scenarios as part of the GARTEUR FM-AG(16) aircraft simulation benchmark. The study resulted in high-fidelity nonlinear fault models for a civil large transport aircraft that addresses the need to improve the prediction of reconfigurable system performance in degraded modes.

\section{FLIGHT 1862 AIRCRAFT ACCIDENT CASE}

On October 4, 1992, a Boeing 747-200F freighter aircraft, Flight 1862 (Fig. 2), went down near Amsterdam Schiphol Airport after the separation of both rightwing engines. In an attempt to return to the airport for an emergency landing,

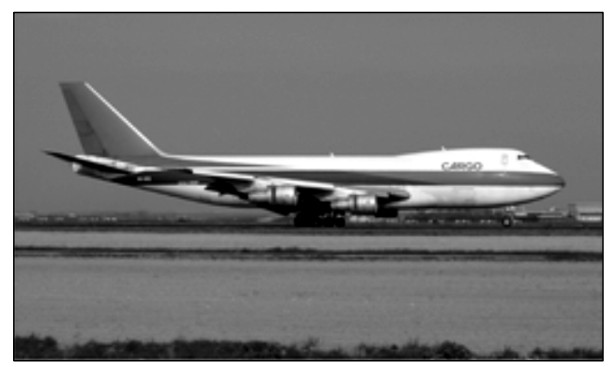

(a)

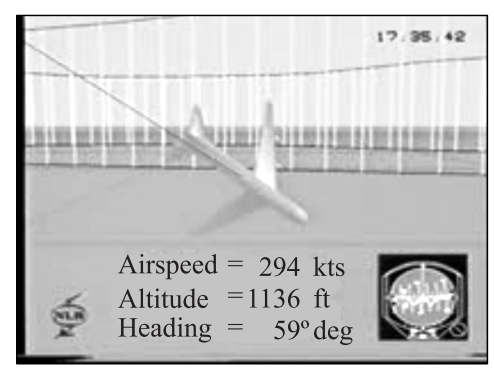

(b)

Figure 2 Cargo accident aircraft prior to takeoff at Amsterdam Schiphol Airport (a); and reconstructed loss of control based on flight data following separation of the rightwing engines (b), EL AL Flight 1862, B747-200F, Amsterdam, 1992 
the aircraft flew several right-hand circuits in order to lose altitude and to line up with the runway as intended by the crew. During the second line-up, the crew lost control of the aircraft. As a result, the aircraft crashed, $13 \mathrm{~km}$ east of the airport, into an eleven-floor apartment building in the Bijlmermeer, a suburb of Amsterdam. Results of the accident investigation, conducted by several organizations including the Netherlands Accident Investigation Bureau and the aircraft manufacturer, were hampered by the fact that the actual extent of the structural damage to the right-wing, due to the loss of both engines, was unknown. The analysis from this investigation concluded that given the performance and controllability of the aircraft after the separation of the engines, a successful landing was highly improbable [10].

In 1997, the division of Control and Simulation of the Faculty of Aerospace Engineering of DUT, in collaboration with the Netherlands National Aerospace Laboratory NLR, performed an independent analysis of the accident [10]. In contrast to the analysis performed by the Netherlands Accident Investigation Bureau, the parameters of the DFDR were reconstructed using comprehensive modeling, simulation, and visualization techniques. In this alternative approach, the DFDR pilot control inputs were applied to detailed flight control and aerodynamic models of the accident aircraft. The purpose of the analysis was to acquire an estimate of the actual flying capabilities of the aircraft and to study alternative (unconventional) pilot control strategies for a successful recovery. The application of this technique resulted in a simulation model of the impaired aircraft that could reasonably predict the performance, controllability effects, and control surface deflections as observed on the DFDR. The analysis of the reconstructed model of the aircraft, as used for the GARTEUR FM-AG(16) benchmark, indicated that from a flight mechanics point of view, the Flight 1862 accident aircraft was recoverable if unconventional control strategies were used [10].

\subsection{Aircraft Damage Configuration}

The Flight 1862 damage configuration to both the aircraft's structure and onboard systems, after the separation of both right-wing engines, is illustrated in Fig. 3. An analysis of the engine separation dynamics concluded that the sequence was initiated by the detachment of the right inboard engine and pylon (engine No.3) from the main wing due to a combination of structural overload and metal fatigue in the pylon-wing joint. Following detachment, the right inboard engine struck the right outboard engine (engine No. 4) in its trajectory also rupturing the right-wing leading edge up to the front spar. The associated loss of hydraulic systems resulted in limited control capabilities due to unavailable control surfaces aggravated by aerodynamic disturbances caused by the right-wing structural damage. 


Alight 1862 Failure Mode Configuration
Aircraft systems
Hydraulic systems $\# 3$ and $\# 4$ lost
Engines \#3 and \#4 thrust loss
Lower rudder lag
Mass properties
Engines \#3 and \#4 weight loss, $4.014 \mathrm{~kg}$ each
Pylon \#3 and \#4 weight loss, $\pm 1.000 \mathrm{~kg}$ each
Lateral center of gravity displacement
Total weight loss: $10.0028 \mathrm{~kg}$
Aerodynamics
Lift loss due to wing damage $\left(\Delta L_{\text {wing }}\right)$
Rolling moment due to wing damage $\left(\Delta \bar{L}_{\text {wing }}\right)$
Drag due to wing damage $\left(\Delta D_{\text {wing }}\right)$
Yawing moment due to wing damage $\left(\Delta N_{\text {wing }}\right)$
Pitching moment due to wing damage $\left(\Delta M_{\text {wing }}\right)$
Right inboard aileron and spoiler $\# 10$ and $\# 11$
aerodynamic efficiency loss

Figure 3 Failure modes and structural damage configuration of the Flight 1862 accident aircraft suffering right-wing engine separation, partial loss of hydraulics, and change in aerodynamics

The crew of Flight 1862 was confronted with a flight condition that was very different from what they expected based on training. The Flight 1862 failure mode configuration resulted in degraded flying qualities and performance that required adaptive and unconventional (untrained) control strategies. Additionally, the failure mode configuration caused an unknown degradation of the nominal flight envelope of the aircraft in terms of minimum control speed and manoeuvrability. For the heavy aircraft configuration at a relative low speed of around 260 knot IAS (indicated airspeed), the DFDR indicated that flight control was almost lost requiring full rudder pedal, 60 to 70 percent maximum control wheel deflection and a high thrust setting on the remaining engines.

\subsection{Aircraft Survivability Assessment}

Figure 4 presents the performance capabilities of the Flight 1862 accident aircraft after separation of both right-wing engines, reconstructed via the methods described in [10], as a function of thrust and aircraft weight. The reconstructed model indicates that in these conditions and at heavy weight $(700,000 \mathrm{lbs} /$ $317,460 \mathrm{~kg}$ ), level flight capability was available between Maximum Continuous 


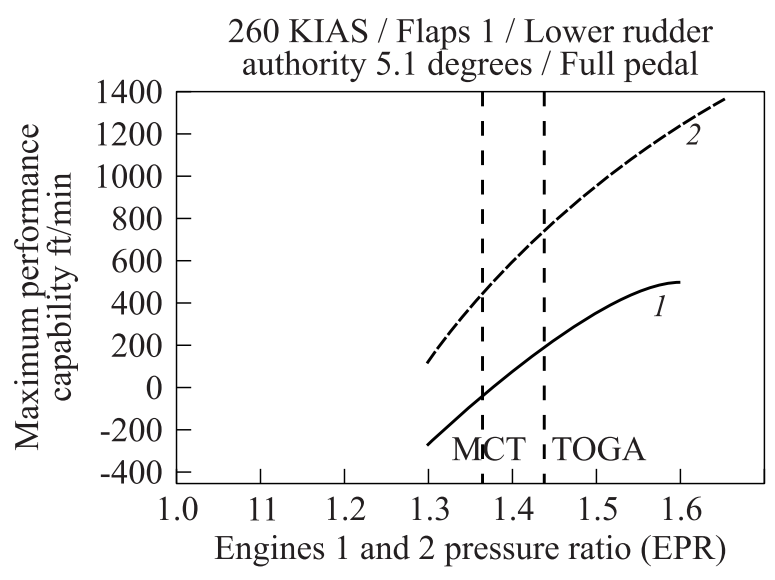

Figure 4 Flight 1862: effect of engine thrust and weight on maximum climb performance for straight flight at 260 knot IAS: $1-700,000 \mathrm{lbs} / 317,460 \mathrm{~kg}$; and $2-$ $577,648 \mathrm{lbs} / 261,972 \mathrm{~kg}$

Thrust (MCT) and Takeoff/Go Around thrust (TOGA). At or above approximately TOGA thrust, the aircraft had limited climb capability. Analysis shows that adequate control capabilities remained available to achieve the estimated performance capabilities. Figure 4 indicates a significant improvement in available performance and controllability at a lower weight if more fuel had been jettisoned.

Simulation analysis of the accident flight using the reconstructed model [10] predicts sufficient performance and controllability, after the separation of the engines, to fly a low-drag approach profile at a 3.5 degree glide slope angle for a high-speed landing or ditch at 200/210 knot IAS and at a lower weight. Note again that this lower weight could have been obtained by jettisoning more fuel. The lower thrust requirement for this approach profile results in a significant improvement in lateral control margins that are adequate to compensate for additional thrust variations. The above predictions have been confirmed during the piloted simulator campaign later in the FM-AG(16) program.

\section{GARTEUR RECOVER BENCHMARK}

For the assessment of novel FTFC techniques, the GARTEUR FM-AG(16) developed a simulation benchmark, based on the reconstructed Flight 1862 aircraft model (RECOVER). The benchmark simulation environment is based on the Delft University Aircraft Simulation and Analysis Tool DASMAT. The DASMAT 
tool was further enhanced with a full nonlinear simulation of the Boeing 747100/200 aircraft (Flightlab747/FTLAB747), including flight control system architecture, for the Flight 1862 accident study as conducted by Delft University. The simulation environment was subsequently utilized and further enhanced as a realistic tool for evaluation of fault detection and fault tolerant control schemes within other research programmes [11].

The GARTEUR RECOVER benchmark has been developed as a Matlab ${ }^{\circledR /}$ Simulink ${ }^{\circledR}$ platform for the design and integrated (real-time) evaluation of new fault tolerant control techniques. The benchmark consists of a set of high-fidelity simulation and flight control design tools, including aircraft fault scenarios validated against accident flight data. The GARTEUR RECOVER benchmark software package is equipped with several simulation and analysis tools, all centered around a generic nonlinear aircraft model for six degrees-of-freedom nonlinear aircraft simulations. For high performance computation and visualization capabilities, the package has been integrated as a toolbox in the computing environment Matlab ${ }^{\circledR} /$ Simulink $^{\circledR}$. The tools of the GARTEUR RECOVER benchmark include trimming and linearization for (fault tolerant) flight control law design, nonlinear offline (interactive) simulations, simulation data analysis, and flight trajectory and pilot interface visualizations (Fig. 5). The modularity of the benchmark makes it customisable to address research goals in terms of aircraft type, flight control system configuration, failure scenarios, and assessment criteria. The GARTEUR RECOVER benchmark is operated via a Matlab ${ }^{\circledR}$ graphical user interface (see Fig. 5) from which the different benchmark tools may be selected. The user options in the main menu are divided into three main sections allowing to initialize the benchmark, run the simulations, and select the analysis tools.

The test scenarios that are an integral part of the GARTEUR RECOVER benchmark were selected to provide challenging (operational) assessment criteria, as specifications for reconfigurable control, to evaluate the effectiveness and potential of the FTFC methods being investigated. Validated against data from the DFDR, the benchmark provides accurate failure models, realistic scenarios, and assessment criteria for a civil large transport aircraft with fault conditions ranging in severity from major to catastrophic.

The geometry of the GARTEUR RECOVER benchmark flight scenario is roughly modeled after the Flight 1862 accident profile (Fig. 6). The scenario consists of a number of phases. First, it starts with a short section of normal flight after which a fault occurs, which is, in turn, followed by a recovery phase. If this recovery is successful, the aircraft should again be in a stable flight condition, although not necessarily at the original altitude and heading. After recovery, an optional identification phase is introduced during which the flying capabilities of the aircraft can be assessed. This allows for a complete parameter identification of the model for the damaged aircraft as well as the identification of the safe flight envelope. Hopefully, the knowledge gained during 


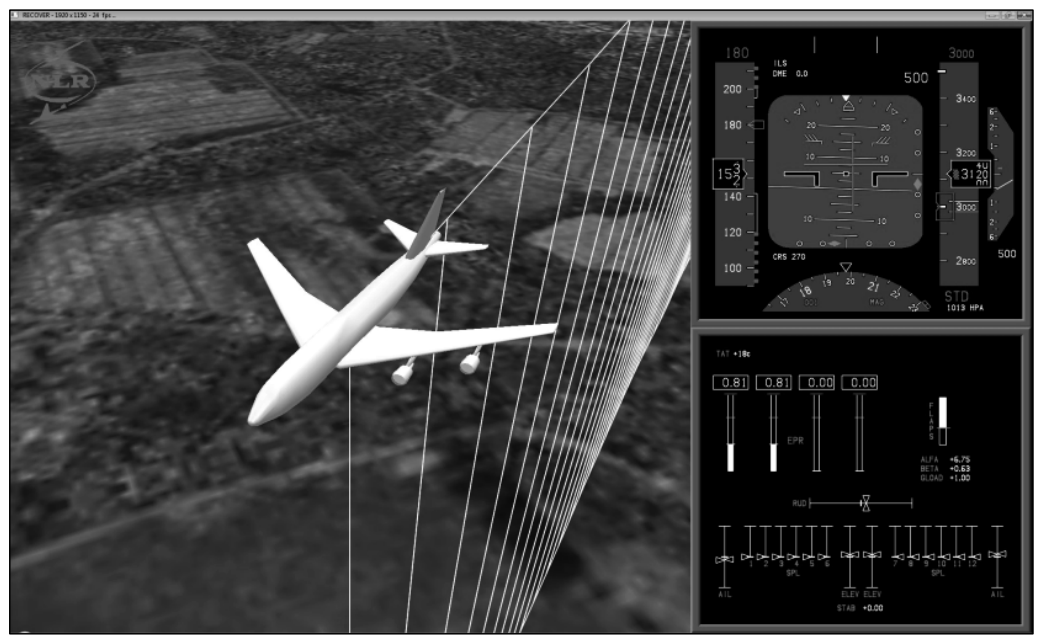

(a)

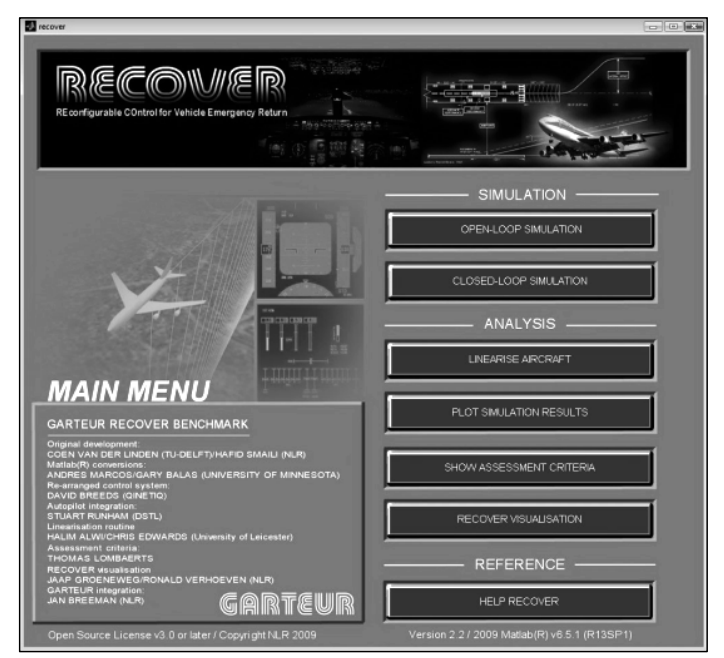

(b)

Figure 5 GARTEUR RECOVER benchmark high-resolution aircraft visualization for interactive (real-time) simulation of new fault tolerant flight control algorithms $(a)$; and RECOVER benchmark main menu for selection of simulation and analysis tools $(b)$ 


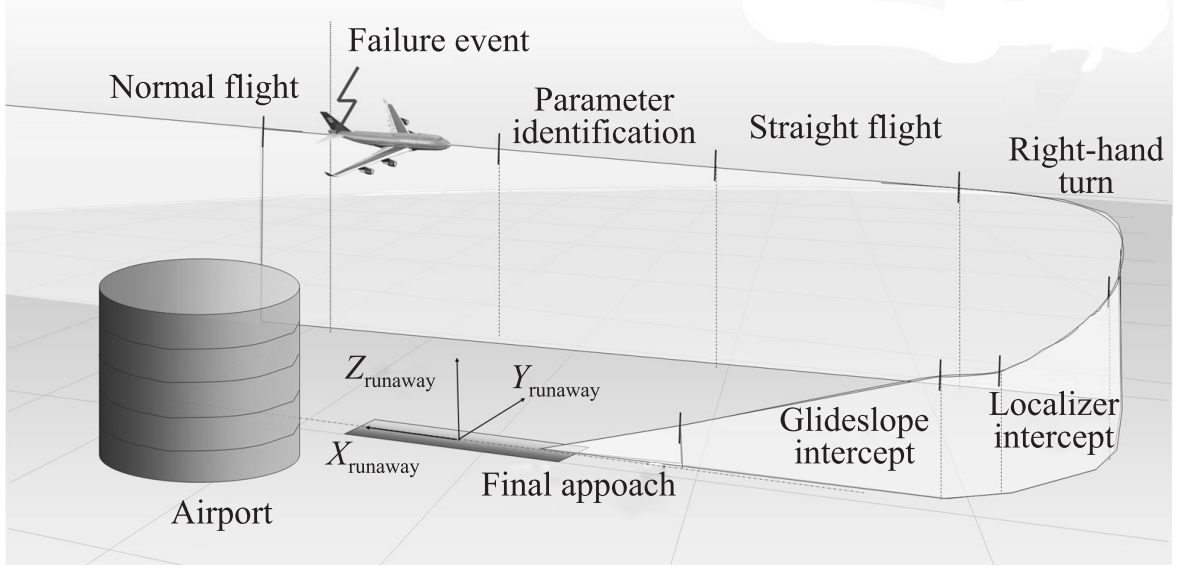

Figure 6 GARTEUR FM-AG(16) benchmark scenario representing standard terminal approach profile for qualification of FTFC strategies for safe landing of a damaged transport aircraft

this identification phase can be used by the controller to improve the chances of a safe landing. In principle, the flight control system is now reconfigured to allow safe flight. The performance of the reconfigured aircraft is subsequently assessed in a series of five flight phases. These consist of straight and level flight, a right-hand turn to a course intercepting the localizer, localizer intercept, glideslope intercept, and the final approach. During the final approach phase, the aircraft is subjected to a sudden lateral displacement just before the threshold, which simulates the effect of a low altitude windshear. The landing itself is not a part of the benchmark, because a realistic aerodynamic model of the damaged aircraft in ground effect is not available. However, it is believed that if the aircraft is brought to the threshold in a stable condition, the pilot will certainly be able to take care of the final flare and landing.

\section{FLIGHT SIMULATOR INTEGRATION AND PILOTED ASSESSMENT}

The developed FTFC schemes in this project have been evaluated in a piloted simulator assessment using a real-time integration of the GARTEUR RECOVER benchmark model, including reconstructed accident scenarios $[2,3]$. The evalua- 


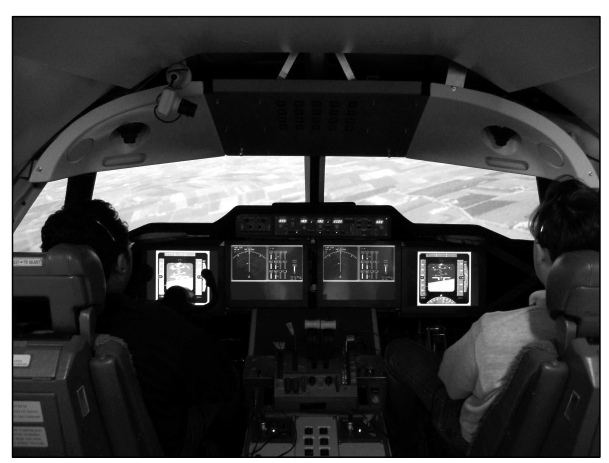

(a)

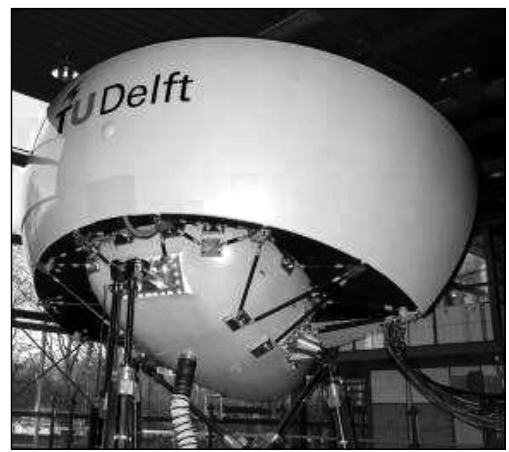

(b)

Figure 7 Evaluation of GARTEUR FM-AG(16) FTFC techniques in the Delft University SIMONA Research Simulator based on reconstructed accident scenarios: $(a)$ Boeing 747 cockpit configuration; and (b) visual system dome

tion was conducted in the SIMONA Research Simulation (SRS) facility, a full 6 degree-of-freedom motion research simulator, of DUT (Fig. 7).

Several validation steps were performed to assure the Benchmark model was implemented correctly. This included proof-of-match validation and piloted checkout of the baseline aircraft, control feel system, and Flight 1862 controllability and performance characteristics. To accurately replicate the operational conditions of the reconstructed Flight 1862 accident aircraft in the simulator, the experiment scenario was aimed at a landing on runway 27 of Amsterdam Schiphol airport. The SIMONA airport scenery was representative of Amsterdam Schiphol airport and its surroundings for flight under visual flight rules (VFR).

The GARTEUR FM-AG(16) piloted simulator campaign provided a unique opportunity to assess pilot performance under flight validated accident scenarios and operational conditions. Six professional airline pilots, with an average experience of about 15.000 flight hours, participated in the piloted simulations. Five pilots were type rated for the Boeing 747 aircraft while one pilot was rated for the Boeing 767 and Airbus A330 aircraft.

In general, the results show, for both automatic and manual controlled flight, that the developed FTFC strategies were able to cope with potentially catastrophic failures in case of flight critical system failures or if the aircraft configuration has changed dramatically. In most cases, apart from any slight failure transients, the pilots commented that aircraft behavior felt conventional after control reconfiguration following a failure, while the control algorithms were successful in recovering the ability to control the damaged aircraft. Manual controlled flight under fault reconfiguration was assessed for both a runaway of 


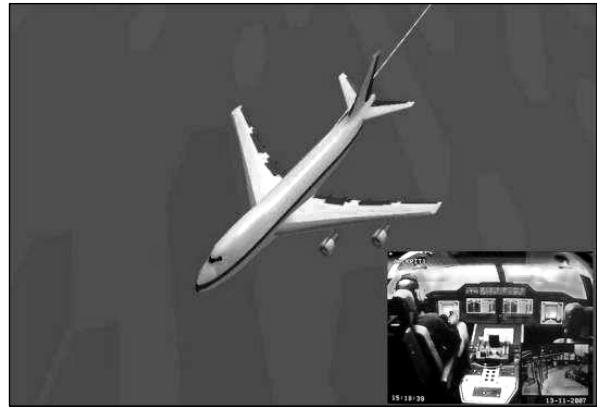

(a)

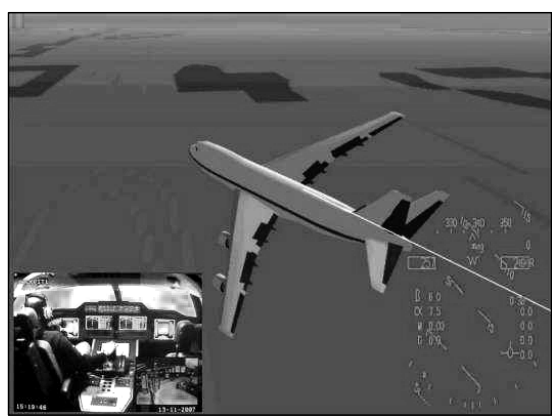

(b)

Figure 8 The FM-AG(16) piloted simulation showing the reconstructed Flight 1862 accident aircraft with separated right-wing engines $(a)$; and piloted simulation showing a sudden hardover of the rudder inducing a large roll upset of the aircraft without reconfigurable control laws (b) (flight animation by Rassimtech AVDS ${ }^{\circledR}$ )

the rudder to the blowdown limit and a separation of both right-wing engines (Fig. 8). Part of the FTFC strategies that were evaluated in the piloted simulation campaign consisted of a combination of real-time aerodynamic model identification and adaptive nonlinear dynamic inversion for control allocation and reconfiguration $[1,2]$. The simulation results have shown that the handling qualities of the reconfigured damaged aircraft with a fault tolerant control system degrade less, indicating improved task performance. For both the Flight 1862 and rudder hardover case, as part of the scenarios surveyed in this research programme, the pilots demonstrated the ability to fly the damaged aircraft, following control reconfiguration, back to the airport and conduct a survivable approach and landing.

\section{SUMMARY AND CONCLUDING REMARKS}

A benchmark for the integrated evaluation of new fault detection, isolation, and reconfigurable control techniques has been developed within the framework of the European GARTEUR FM-AG(16) on Fault Tolerant Control. Validated against data from the DFDR, the benchmark addresses the need for high-fidelity nonlinear simulation models to improve the prediction of reconfigurable system performance in degraded modes. The GARTEUR RECOVER benchmark is suitable for both offline design and analysis of new FTFC system algorithms and integration on simulation platforms for piloted hardware in the loop testing. In conjunction with enhanced graphical tools, including high resolution aircraft visualizations, the benchmark supports tool-based advanced flight con- 
trol system design and evaluation within research, educational, or industrial framework.

The GARTEUR FM-AG(16) on Fault Tolerant Control has made a significant step forward in terms of bringing novel 'intelligent' self-adaptive flight control techniques, originally conceived within the academic and research community, to a higher technology readiness level. The research programme demonstrated that the designed fault tolerant control algorithms were successful in recovering control of significantly damaged aircraft.

Within the international aviation community, urgent measures and interventions are being undertaken to reduce the amount of loss of control accidents caused by mechanical failures, atmospheric events, or pilot disorientation. The application of fault tolerant and reconfigurable control, including aircraft envelope protection, has been recognized as a possible long-term option for reducing the impact of flight critical system failures, pilot disorientation following upsets, or flight outside the operational boundaries in degraded conditions (e.g., icing). Fault tolerant flight control, and the (experimental) results of this Action Group, may further support these endeavors in providing technology solutions aiding the recovery and safe control of damaged aircraft or in-flight upset conditions. Several organizations within this Action Group currently conduct in-flight loss of control prevention research within the EC Framework 7 programme Simulation of Aircraft Upsets in Aviation SUPRA (www.supra.aero). The experience obtained by the partners in this Action Group will be utilized to study future measures in mitigating the problem of in-flight loss of control and upset recovery and prevention.

The results of this research programme on FTFC, as described in this paper, have been published in the book Fault tolerant flight control - a Benchmark challenge by Springer-Verlag (2010) under the Lecture notes in control and information sciences series (LNCIS-399). The GARTEUR RECOVER benchmark simulation model, accompanying the book, is available via the project's website (www.faulttolerantcontrol.nl) after registration. The website provides further access to contact information, follow-on projects, and future software updates.

\section{ACKNOWLEDGMENTS}

The authors recognize the contributions of the members of the GARTEUR FMAG(16) to this paper. The authors also appreciate the funding that the Dutch Technology Foundation STW has provided as a part of the GARTEUR activities. Special thanks to Jaap Groeneweg and Ronald Verhoeven of NLR for their contribution to the RECOVER aircraft visualization tools. Finally, a word of thanks to all those who have contributed to the further improvement of the GARTEUR 
RECOVER benchmark model within their flight control research programmes, especially Andres Marcos of DEIMOS Space and Gary Balas of the University of Minnesota.

\section{REFERENCES}

1. Lombaerts, T. J. J., P. Chu, J. A. Mulder, and D. A. Joosten. 2009. Flight control reconfiguration based on a modular approach. IFAC SAFEPROCESS 2009 Conference Proceedings. Barcelona, Spain.

2. Edwards, C., T. J. J. Lombaerts, and M. H. Smaili. 2010. Fault tolerant control a benchmark challenge. Lecture notes in control and information sciences ser. (LNCIS-399). Springer-Verlag.

3. Stroosma, O., M.H. Smaili, and J. A. Mulder. 2009. Pilot-in-the-loop evaluation of fault tolerant flight control systems. IFAC SAFEPROCESS 2009 Conference Proceedings. Barcelona, Spain.

4. Civil Aviation Authority of the Netherlands (CAA-NL). 2007. Civil aviation safety data.

5. NTSB. 1999. Uncontrolled descent and collision with terrain USAir Flight 427 Boeing 737-300, N513AU near Aliquippa, Pennsylvania September 8, 1994. National Transportation Safety Board, Aircraft Accident Report NTSB/AAR-99/01.

6. NTSB. 2001. Uncontrolled descent and collision with terrain United Airlines Flight 585 Boeing 737-200, N999UA 4 miles south of Colorado Springs Municipal Airport Colorado Springs, Colorado March 3, 1991. National Transportation Safety Board, Aircraft Accident Report NTSB/AAR-01/01.

7. Sogame, H. 1993. Lauda Air Luftfart Aktiengesellschaft Boeing 767-300ER registration OE-LAV Dan Chang District Suphan Buri Province Thailand 26 May 1991. Aircraft Accident Investigation Committee Ministry of Transport and Communications Thailand.

8. NTSB. 1990. United Airlines Flight 232 McDonnell Douglas DC-10-10 Sioux Gateway Airport Sioux City, IOWA July 19, 1989. National Transportation Safety Board, Aircraft Accident Report NTSB/AAR-90/06.

9. Netherlands Aviation Safety Board. 1994. EL AL Flight 1862. Aircraft Accident Report 92-11. Hoofddorp.

10. Smaili, M. H., and J.A. Mulder. 2000. Flight data reconstruction and simulation of the 1992 Amsterdam Bijlmermeer airplane accident. AIAA PaperNo. 2000-4586.

11. Marcos, A., and G. J. Balas. 2001. Linear parameter varying modeling of the Boeing 747-100/200 longitudinal motion. AIAA Paper No. 2001-4347. 